\title{
La calidad de muerte como componente de la calidad de vida
}

María Ángeles Durán

Consejo Superior de Investigaciones Científicas

RESUMEN

La aspiración a una buena calidad de vida se prolonga de modo natural en la aspiración a una buena calidad de muerte. En este estudio se sintetizan una docena de estudios sociológicos realizados en España por diversas instituciones a lo largo de la última década (CIS, CIRES y ASEP, entre otras) sobre diversos aspectos de la muerte, el testamento vital y la eutanasia. La conclusión principal es que las opiniones y actitudes de la sociedad española en este tema son mucho más abiertas que lo reconocido por la legislación vigente. El tema es de gran interés y actualidad, por su aparición en el programa electoral del partido que ganó las elecciones en los comicios de marzo de 2004 y por las propuestas legislativas en curso.

Palabras clave: Muerte, Mortalidad, Eutanasia, España. 
Este trabajo es continuación de algunos trabajos míos anteriores de sociología de la salud $^{1}$, de los que los principales exponentes son los libros Desigualdad social y enfermedad $(1983)^{2}$ y Los costes invisibles de la enfermedad $(2003)^{3}$, así como el artículo «El coste de la salud para las familias» $(2003)^{4}$. No obstante, hay que destacar que probablemente no habría llegado a escribirlo si al interés científico y social por la situación de los enfermos graves y terminales no se le hubiera sumado mi propia experiencia personal de la enferme$\mathrm{dad}^{5}$, que me hizo ver la urgente necesidad de mayor investigación sobre el modo en que los enfermos, sus familiares y el personal sanitario se enfrentan al momento cercano de la muerte.

La primera versión de este artículo fue realizada a petición de la International Society for Quality-Of-Life-Studies (ISQOLS), y fue presentada en una conferencia abierta al público durante su VI Conference, celebrada en la Goëthe Universitat, Frankfurt, 20-24 de julio de 2003.

Agradezco al Centro de Investigaciones Sociológicas (CIS), Centro de Investigaciones sobre la Realidad Social (CIRES) y a Análisis Sociales, Económicos y Políticos (ASEP) las facilidades recibidas para la utilización de sus bancos de datos.

\section{DE LA CANTIDAD A LA CALIDAD}

De acuerdo con las más recientes publicaciones de Eurostat, en la EU-15 tienen lugar 3,7 millones de muertes cada año, de las que 358.000 corresponden a España. La muerte es uno de los escasos acontecimientos de los que podemos tener certeza que nos van a suceder. Sin embargo, la muerte es una rara e infrecuente circunstancia; sólo afecta a algo menos de diez de cada mil personas cada año, y sucede sobre todo a personas de edad muy avanzada, de más de ochenta años, que tienen escasas oportunidades de luchar para organizarse por sí mismos y mejorar sus condiciones de vida y muerte. Las causas principales de muerte en los países desarrollados son las enfermedades circulatorias, el

\footnotetext{
1 Agradezco al Plan Nacional de I+D+l (2000-2003) la concesión del proyecto El uso del tiempo: integración en el análisis de la estructura social y económica, Ref. SEC2002-00504.

2 M. A. Durán: Desigualdad social y enfermedad, Tecnos, Madrid, 1983.

3 M. A. Durán: Los costes invisibles de la enfermedad, Fundación BBVA, Madrid, 2003.

4 M. A. Durán: «El coste de la salud para las familias», revista Arbor, n. ${ }^{\circ}$ 694, 2003.

5 Esta experiencia ha sido relatada, de modo autobiográfico, en el libro Diario de Batalla. Mi lucha contra el cáncer, Aguilar, Madrid, 2003.
} 
cáncer, las enfermedades respiratorias y otras varias causas, que incluyen accidentes y suicidios. Pero las causas varían significativamente según edad, sexo y estilo de vida. En 1998, las tasas standard de muerte por 100.000 personas en la Europa de los Quince eran 908,5 para los varones, en tanto que sólo alcanzaban 537,8 para las mujeres, un tercio menos que los hombres. A pesar del constante incremento en la esperanza de vida, y debido precisamente al envejecimiento de la población, las cifras relativas de la mortalidad respecto a la población total ya no mejoran en Europa, ni es previsible que mejoren a corto o medio plazo.

Durante el siglo $x x$, algunos grandes cambios tecnológicos y sociales permitieron la modificación de los bordes naturales de la vida: nacimiento y muerte. Por lo que se refiere al nacimiento, en todos los países desarrollados los cambios trajeron consigo nuevos modos de definir la «calidad de nacimiento», que en la actualidad incluye cuatro aspectos básicos: libertad de decisión, bajo riesgo para el niño y la madre, confort y ausencia de dolor en la gestación y parto, así como cobertura económica y social tanto para el niño como para sus progenitores.

Respecto a la segunda frontera de la vida, la muerte, han tenido lugar pocos cambios prácticos. El único gran cambio que realmente ha tenido lugar es su aplazamiento, el incremento general de la esperanza de vida. Sin embargo, la opinión pública está cambiando realmente respecto a este tema. En dos países europeos (Holanda y Bélgica), ya se han aprobado cambios legales que reconocen la autonomía de los pacientes terminales para decidir sobre su propia muerte. Lo mismo ha sucedido en el Estado norteamericano de Oregón, cuyaLey de Eutanasia ha sido reforzada por una reciente sentencia del Tribunal Federal de Apelaciones, si bien esta última no se refiere a asuntos de fondo, sino a los procedimientos. En España, algunos tímidos avances legales en el reconocimiento de la autonomía del sujeto en relación con la muerte se produjeron en Cataluña, Extremadura, Galicia y País Vasco. Una proposición de Ley de disponibilidad de la propia vida, presentada por el Grupo Parlamentario de Izquierda VerdeIzquierda Unida-Iniciativa per Catalunya Verds, ha sido publicada en el Boletín Oficial de las Cortes Generales el 23 de abril de 2004. El Observatori de Bioética i Dret, de Barcelona, ha elaborado en el año 2003 un Documento sobre la disposición de la propia vida en determinados supuestos; declaración sobre la eutanasia. El documento, difundido a través de Internet y presentaciones públicas, incluye recomendaciones para la actuación de los poderes públicos ( «se ha de introducir una legislación específica que permita la práctica de la eutanasia en cuanto acción médica a petición seria, expresa, inequívoca y reiterada de la persona afectada»), del personal sanitario, del uso de los documentos de Voluntades Anticipadas, autonomía relativa de los menores y cuidados paliativos. 
Probablemente, el siglo XXI conocerá cambios similares en las actitudes respecto a la muerte de los que vivió el siglo xx respecto al nacimiento (M. Castra, 20036; D. Thomasma y T. Kushner, 19997; C. G. Prado y S. J. Taylor, $1999^{8}$ ).

Este trabajo analizará las condiciones del «modelo ideal de muerte», tal como ha sido estudiado en algunas muestras de población en España por los profesores Jesús de Miguel y M. Marí-Klose, así como los resultados de una docena de encuestas hechas en España sobre la frecuencia de pensamiento sobre la muerte, preocupaciones que la acompañan y actitudes acerca de la intervención en el caso de los enfermos terminales que la solicitan. Estos resultados se analizan en el contexto de una sociedad que cada vez se va haciendo más y más aconfesional, y que crecientemente reconoce el respeto a la autonomía personal de los sujetos durante toda su vida. Las asociaciones de enfermos y los familiares de enfermos pueden jugar un importante papel como agentes de cambio social, modificando las condiciones de muerte. Esto es especialmente probable en el caso de enfermedades como el cáncer, en las que la muerte puede conocerse con bastante anticipación y cuando tanto los pacientes como sus familias y amigos aún se encuentran en una edad socialmente activa que les permite luchar por sus propios ideales. También son importantes agentes de cambio las asociaciones internacionales del tipo Federación Mundial de Asociaciones pro Derecho a Morir, en la que se encuentran federadas treinta y siete asociaciones nacionales. La asociación DMD (Derecho a Morir Dignamente) realizó en 2003 un sondeo entre sus socios respecto a la eutanasia, cuyos resultados han sido analizados por Salvador Giner y difundidos por la asociación. La muestra recogida es poco representativa desde el punto de vista estadístico, pero es una buena aportación al tema por sus aspectos cualitativos.

\section{LA PRESENCIA DE LA MUERTE EN LA VIDA COTIDIANA}

La frecuencia de pensamiento sobre la muerte es un índice del grado de presencia de este tema en la vida cotidiana. Los datos presentados en la tabla 1 se obtuvieron en una encuesta de ámbito nacional realizada en España en el año 2002, acerca de varios temas de opinión pública. La muestra fue de 2.500 entrevistas personales a población mayor de dieciocho años, y fue realizada por el Centro de Investigaciones Sociológicas (CIS).

\footnotetext{
6 M. Castra (2003): Bien mourir. Sociologie des soins palliatifs, Le Lien social, Presses Universitaires de France, París.

7 D. Thomasma y T. Kushner (eds.) (1999): De la vida a la muerte. Ciencia y bioética, Editorial Cambridge, Madrid.

8 C. G. Prado y S. J. Taylor (1999): Assisted Suicide: Theory and Practice in Elective Death, Humanity Books, New York.
} 
TABLA 1

Frecuencia de pensamiento sobre la muerte por edad (en porcentajes)

\begin{tabular}{llrrr} 
A & Frecuencia & Total & 18-24 años & 65 y más \\
\hline 4 & Muy frecuente & 14,1 & 9,2 & 24,7 \\
3 & Algunas veces & 41,7 & 36,2 & 42,1 \\
2 & Casi nunca & 25,2 & 30,4 & 15,9 \\
1 & Nunca & 18,6 & 23,9 & 16,3 \\
$-\quad$ Sin respuesta & 0,5 & 0,3 & 1,0 \\
\hline & Total & 100,0 & 100,0 & 100,0 \\
& Media & 2,32 & 2,06 & 2,73
\end{tabular}

FUENTE:

Centro de Investigaciones Sociológicas, España, 2002. Encuesta nacional, 2.500 entrevistas personales a población mayor de 18 años. Número de Estudio: 2442. La media se obtiene ponderando las respuestas con la escala A.

La primera conclusión que podría obtenerse de la encuesta es que la muerte no es un motivo frecuente de pensamiento. Sólo el $14,1 \%$ de la población le dedica pensamientos muy frecuentes y el $18,6 \%$ nunca piensa en ella. No obstante, si se añaden las respuestas «frecuentemente» y «algunas veces», el conjunto de los que le dedican algún tiempo de pensamiento cubre el $55,8 \%$ de la población, esto es, la mayoría.

La edad es una variable asociada con la frecuencia de pensamiento sobre la muerte. En el grupo más joven de la muestra (los que tienen entre 18 y 24 años), sólo el 9,2\% piensa en ello frecuentemente, y el $23,9 \%$ no lo hace nunca. En contraste, en el grupo de edad de más de 65 años, el porcentaje de quienes piensan frecuentemente en la muerte es casi tres veces más alto que entre los jóvenes. El índice sintético da como resultado 2,32 puntos para la población general, 2,06 para los jóvenes y 2,73 para los mayores. La acumulación de experiencias relacionadas con la muerte (la muerte de los propios padres, la de los familiares y amigos), así como la aproximación a la edad que marca el final de la esperanza de vida, el paso por otros umbrales vitales tales como la jubilación, o la experiencia de los propios achaques y enfermedades, favorecen la reflexión sobre la muerte. En el grupo de los mayores de 65 años, más de dos tercios de los entrevistados declaran que piensan en la muerte frecuentemente 0 , al menos, algunas veces.

Las mujeres son más longevas que los varones, y justamente esta diferencia de edad podría explicar que piensen más que los varones en la muerte (tabla 2). Sin embargo, la 
diferencia entre ambos grupos es demasiado elevada como para deberse solamente a la experiencia personal del envejecimiento. También revela un patrón cultural y estructural de relaciones con la muerte, tanto la propia muerte como la de los demás, que es diferente para hombres y mujeres. A las mujeres les afecta más la muerte porque no sólo tienen que encarar la suya propia, sino la de sus más próximos familiares y amigos. Son ellas quienes se ocupan principalmente de los enfermos y de los que van a morir, quienes les acompañan y cuidan durante la enfermedad, la vejez, la discapacidad y los momentos finales de la vida. Además, también les corresponden socialmente la mayor parte de las actividades relacionadas con los procesos de duelo post-mortem: el enterramiento, funerales y honras fúnebres, así como el mantenimiento del recuerdo y la memoria colectiva.

La tarea de hacerse cargo de la muerte de los otros no es sencilla: todos nosotros podemos recordar con los ojos cerrados el patetismo de algunas imágenes universales, como la magnífica Pietá de Miguel Ángel que se guarda en el Vaticano, en la que María soporta sobre sus rodillas y brazos el cuerpo muerto de Jesús. O, en otro orden de interpretación literaria, el mito griego de Antígona, en el que la joven mujer protagonista va a la muerte en la flor de su edad porque no puede resolver el conflicto entre el deber de lealtad para con su hermano fallecido (enterrarle y honrarle) y el deber de lealtad a las leyes políticas de su ciudad, que se lo prohíben.

Incluso desde la perspectiva social y económica, las mujeres resultan más afectadas por el fallecimiento de sus compañeros varones que a la inversa. Las mujeres dependen más de los ingresos de sus compañeros (breadwinners, en terminología inglesa). Como generalmente son más jóvenes que sus esposos, la probabilidad de vivir en carne propia la experiencia de la muerte del otro y la consiguiente viudez es muy elevada.

En cuanto a la pre-muerte, no es una etapa definida solamente por consideraciones médicas; también definen este período la atmósfera cultural y las creencias particulares de cada persona. Para algunas corrientes filosóficas, el período de pre-muerte es tan largo como la vida misma, porque, en su interpretación de la vida, el punto de mira es precisamente la muerte, el final. Según estas creencias, la verdadera vida es la sobrenatural, y sólo comienza en el momento en que la vida terrenal y mortal toca a su fin.

En cambio, para otras corrientes filosóficas o posiciones vitales, la muerte es vista desde una perspectiva opuesta, solamente como el final de lo que realmente importa. Según estas creencias, la muerte debería dominarse y controlarse para adaptarla a los mismos valores (libertad, razón ausencia de dolor, incluso alegría) que deberían presidir cualquier otro período de la vida. 
TABLA 2

Frecuencia de pensamiento sobre la muerte según género (en porcentajes)

\begin{tabular}{llrrr} 
A & Frecuencia & Total & Varones & Mujeres \\
\hline 4 & Muy frecuente & 14,1 & 10,4 & 17,5 \\
3 & Algunas veces & 41,7 & 38,3 & 44,9 \\
2 & Casi nunca & 25,2 & 27,4 & 23,0 \\
1 & Nunca & 18,6 & 23,4 & 14,0 \\
$-\quad$ Sin respuesta & 0,5 & 0,4 & 0,5 \\
\hline & Total & 100,0 & 100,0 & 100,0 \\
& Media & 2,32 & 2,12 & 2,51
\end{tabular}

FUENTE:

Centro de Investigaciones Sociológicas, España, 2002. Encuesta nacional, 2.500 entrevistas personales a población mayor de 18 años. Número de Estudio: 2442. La media se obtiene ponderando las respuestas con la escala A.

Algunas variables sociodemográficas tales como el nivel de educación o el status socioeconómico no presentan una asociación clara con la frecuencia de pensamiento sobre la muerte. Los datos de que disponemos no son suficientemente buenos como para filtrar el efecto que ejercen sobre ellos, una vez desagregada la muestra por grupos de edad. Según estos datos, la clase alta y media-alta manifiestan una frecuencia ligeramente más alta de pensamiento sobre la muerte (15,3 y 14,25\%, respectivamente). Los trabajadores manuales obtienen el porcentaje más alto de respuestas diciendo que «nunca» piensan en la muerte $(22,8 \%$ de los trabajadores cualificados y $19,1 \%$ de los no cualificados).

El pensamiento sobre la muerte no significa necesariamente una preocupación o miedo especial. Incluso entre los mayores, que piensan en la muerte mucho más que los jóvenes, la muerte no es una de sus principales preocupaciones. Dos encuestas del Centro de Investigaciones Sociológicas, una de ellas a la población general (1997) y otra a la población mayor de 65 años (1998), subrayan que este tema no aparece como central entre las preocupaciones manifiestas de los mayores.

En la encuesta general de población (2.500 entrevistas, ámbito nacional, a mayores de 18 años) se preguntó acerca de «las tres preocupaciones principales de las personas mayores». La tabla 3 muestra la imagen proyectada por la población general, así como un extracto de dos segmentos de la población, el más joven y el de edad más avanzada. A pesar 
TABLA 3

Las tres preocupaciones principales de las personas mayores

\begin{tabular}{|c|c|c|c|c|c|}
\hline \multirow[b]{2}{*}{ (Hasta tres respuestas) } & \multirow[b]{2}{*}{ Total } & \multicolumn{3}{|c|}{ Edad } & \multirow{2}{*}{$\begin{array}{l}\text { Género } \\
\text { Mujeres }\end{array}$} \\
\hline & & 18-24 años & 65 y más & Varones & \\
\hline Inactividad & 16,6 & 14,9 & 13,3 & 19,7 & 13,8 \\
\hline Pérdida de memoria & 27,6 & 23,9 & 38,4 & 23,8 & 31,0 \\
\hline Soledad & 76,9 & 78,8 & 71,0 & 75,5 & 78,3 \\
\hline Dependencia & 33,9 & 34,5 & 27,1 & 32,4 & 35,3 \\
\hline Sensación de inutilidad & 32,8 & 41,0 & 21,7 & 33,8 & 31,9 \\
\hline Dolor & 13,3 & 11,7 & 20,2 & 12,7 & 13,9 \\
\hline Deterioro físico & 26,3 & 26,9 & 24,6 & 28,7 & 24,1 \\
\hline Enfermedad & 38,1 & 34,2 & 45,0 & 37,1 & 39,0 \\
\hline Muerte & 13,3 & 17,1 & 12,0 & 14,6 & 12,2 \\
\hline
\end{tabular}

FUENTE:

Centro de Investigaciones Sociológicas, 1997, Encuesta n. ${ }^{\circ}$ 2244, abril 1997, 2.500 entrevistas personales a población en general.

de disponer de tres respuestas posibles, sólo el 13,3\% mencionó la muerte entre las principales preocupaciones de los mayores. Los jóvenes proyectaron una imagen de los mayores como más preocupados por la muerte $(17,1 \%)$ de lo que lo hicieron los propios mayores $(12,0 \%)$. En lugar de ella, fue la soledad la que recibió un número muy alto de menciones en todos los grupos de edad $(76,9 \%)$, algo más elevado entre los más jóvenes que entre los mayores. La enfermedad es el segundo motivo más imaginado como preocupación, aunque más mencionado por los mayores que por los jóvenes. La pérdida de memoria es la tercera causa mencionada por los mayores, pero en cambio no es muy mencionada por los jóvenes. Hay otras dos causas subrayadas por los jóvenes: la dependencia respecto de otras personas y el sentimiento de inutilidad. Probablemente, ambos segmentos de la población perciben de modo diferente la relación intergeneracional; o quizá, simplemente, los mayores perciben su situación del mismo modo que los jóvenes pero le otorgan un valor diferente, esto es, la asumen como un «derecho adquirido» que no les molesta ni preocupa reclamar.

En contraste, el dolor es un motivo de preocupación citado dos veces más por los mayores que por los jóvenes.

El género no marca una diferencia respecto a las preocupaciones mencionadas, o al menos no tanto como la edad. Los varones mencionan algo más frecuentemente la inactividad y el deterioro físico, en tanto que las mujeres mencionan algo más la pérdida de memoria. 
Si ya hemos hecho notar que las mujeres confesaban pensamientos acerca de la muerte con más frecuencia que los varones, ahora deberíamos resaltar que, sin embargo, las mujeres entrevistadas en esta encuesta general de población atribuyen a los mayores algo menos de preocupación acerca de la muerte de la que le atribuyen los varones en la misma encuesta.

Otra encuesta de 1998 permite enriquecer los comentarios sobre la última encuesta mencionada. Los resultados se muestran en la tabla 4. En esta encuesta también se ofrecía respuesta múltiple, aunque solamente con dos respuestas. Se mantuvo el cuestionario utilizado en la encuesta anterior, pero se suprimió la mención explícita a la muerte, así como al deterioro físico y a la inactividad. En cambio, se añadieron como respuestas posibles «la pérdida de amigos», «pérdida de cónyuge o compañero» y «otras causas».

Es de destacar que en ninguna de las dos encuestas se mencionaron explícitamente los problemas económicos o la falta de seguridad, aunque este último aspecto puede formar

Principales preocupaciones de las personas mayores, 1997-1998

\begin{tabular}{lcrr} 
& & A & B \\
\cline { 2 - 4 } & & 1997 & 1998 \\
\cline { 2 - 4 } & Población general & 65 años y más & 65 años y más \\
\hline Pérdida de memoria & 27,6 & 38,4 & 32,9 \\
Soledad & 76,9 & 71,0 & 22,4 \\
Dependencia & 33,9 & 27,1 & 17,1 \\
Sensación de inutilidad & 32,8 & 21,7 & 13,0 \\
Dolor & 13,3 & 20,2 & 14,1 \\
Enfermedad & 38,1 & 45,0 & 47,1 \\
Inactividad & 16,6 & 13,3 & - \\
Deterioro físico & 26,3 & 24,6 & - \\
Muerte & 13,3 & 12,0 & - \\
Pérdida de amigos & - & - & 5,5 \\
Pérdida de esposo o compañero & - & - & 0,5 \\
Otras causas & - & 2,5
\end{tabular}

FUENTES:

A: Centro de Investigaciones Sociológicas, 1997, Encuesta n. ${ }^{\circ}$ 2244, abrill 1997, 2.500 entrevistas personales a población en general.

B: Centro de Investigaciones Sociológicas, 1998, Encuesta n. ${ }^{\circ}$ 2279, marzo 1998, 2.500 entrevistas personales a la población de 65 años y más.

El cuestionario varía ligeramente entre ambas encuestas. 
parte, indirectamente, de la soledad. Lo más interesante de la comparación entre la encuesta de 1997 y la de 1998 es precisamente la reflexión sobre lo mucho que confiamos los investigadores (y el público en general) en lo que preguntamos. Dicho de otro modo, la reflexión sobre las consecuencias sociales de la autoconfianza metodológica. En el plazo de un año es poco probable que las actitudes de la población general sobre estos temas sufran grandes variaciones. Como en la segunda encuesta sólo eran posibles dos respuestas, en el caso de que las respuestas se distribuyesen de modo similar, ambas respuestas tendrían que haber disminuido proporcionalmente un 33\%. Sin embargo, después de que desapareciese explícitamente la respuesta relativa a la muerte como causa de preocupación, nadie pareció sentir la necesidad de tal respuesta, y "otras causas" sólo obtuvo un $2,5 \%$ del total de respuestas.

Parece como si lo que no se menciona en el cuestionario se olvidase. ¿O lo que sucede no es que se olvide, sino solamente que no se responde?

La cuestión tiene trascendencia: tanto en el mundo desarrollado como en el no desarrollado, se mantienen creencias ancestrales, casi mágicas, que atribuyen a las palabras el poder de originar aquello que significan. Consecuentemente, las palabras han de ser manejadas con un cuidado y precaución extremos.

En España, especialmente en algunas regiones, la muerte es todavía una palabra tabú, pervive el uso de numerosos circunloquios y metáforas para evitar citarla. Lo mismo sucede en muchas otras culturas.

En la encuesta de 1998 que estamos comentando, el porcentaje de referencias a la enfermedad se incrementó respecto a la encuesta anterior, probablemente porque recibió algunas de las respuestas que de otro modo habrían podido expresarse como deterioro físico en la encuesta de 1997. La mención a la soledad aumentó considerablemente en la segunda encuesta, revelando que se trata más de una causa de tercer nivel que de una verdadera preocupación de primera magnitud.

Finalmente, no podemos dejar de subrayar que la respuesta «pérdida de amigos» aparece citada once veces más que la «pérdida de cónyuge». ¿A qué se debe? Quizá se deba a que no puede tenerse miedo o preocupación por lo que ya se ha producido, la situación de viudez; o porque el círculo de parientes y amigos incluye a tantas personas que el riesgo de perder a alguno de ellos es casi una certeza. En cualquier caso, éste es un dato sorprendente que invita a la reflexión sobre los límites de la identidad y sobre el papel que el cónyuge y los amigos y demás familiares juegan en la definición de uno mismo y de la calidad de vida. 


\section{EL MODELO DE MUERTE DE «ALTA CALIDAD»}

En el momento actual, y según datos de J. de Miguel, un tercio de la población española fallece en el hospital, a menudo conectada a maquinarias, entubada, aislada y sin posibilidad de hablar con sus familiares. Algunas famosas «malas muertes», que han tenido lugar bajo condiciones de extremo cuidado y atención médica, han impactado a la opinión pública española. Especialmente recordado es el caso de Franco, quien después de gobernar el país durante cuarenta años sufrió una lenta y «mala muerte» que la mayoría de la población hubiera preferido evitar para sí misma o para los seres próximos más queridos. En algunos casos, no infrecuentes, la vida se prolonga bajo condiciones dolorosas y muy onerosas, en las que el conflicto encubierto de intereses dificulta la toma de decisiones por las partes implicadas en el asunto. Para algunos de los implicados, su interés es mantener con vida al paciente mientras utiliza costosas y amplias tecnologías y servicios médicos, aunque en otras ocasiones el interés de estos mismos grupos, dependiendo de la relación económica con el enfermo y sus familiares o aseguradores, sea opuesto al mencionado. El paciente, los profesionales de la salud, los familiares, los contribuyentes que financian el cuidado con sus impuestos, los ideólogos políticos y religiosos, las compañías de seguros, los empleadores, los contrapartes de diversos tipos de contratos de servicios y suministros: todos ellos tienen intereses en la muerte, legítimos pero no siempre coincidentes.

Jesús de Miguel y M. Marí-Klose ${ }^{9}$ han llevado a cabo uno de los pocos estudios empíricos realizados en España sobre el «canon de la muerte», o el modo ideal de morir. Para ello hicieron una serie de pequeñas muestras de entrevistas o pasaron cuestionarios a segmentos variados de población. También utilizaron discusiones de grupo y presentaron resultados, con discusión posterior, ante audiencias de expertos. En cualquier caso, aunque no se trata de una encuesta habitual con muestra al azar representativa, el valor de su aportación deriva esencialmente de la escasez de estudios disponibles. Los rasgos principales de la muerte ideal, o «muerte de alta calidad», revelados por su estudio son los siguientes:
a) Sin dolor.
b) Inconsciente, durmiendo.
c) Rápido, aunque no por accidente.
d) Acompañado por familiares o amigos íntimos.
e) A edad avanzada.
f) En casa.
g) Sin estigma.
h) Con bajo coste para los demás (que no les contagie, lleve a la ruina, etc.).
9 J. de Miguel y M. Marí-Klose (2000): «El canon de la muerte», Política y Sociedad, n. ${ }^{3}$ 35, 2000, pp. 113-144. 
De hecho, no resulta fácil combinar en el mismo caso las circunstancias del tipo b) (inconsciente), c) (rápida) y d) (acompañado). Este último punto puede interpretarse como un rechazo de la llamada «muerte social», que tan a menudo precede a la muerte física y que origina abandono y soledad.

\section{LOS LÍMITES DE LA VOLUNTAD. ASPIRACIONES Y FRONTERAS IMPUESTAS}

Actualmente, el principal debate social sobre la muerte se centra en los límites de la voluntad, en el grado en que se permite intervenir al sujeto y al sistema sanitario en el período previo a la muerte, cuando el desenlace es inminente. Este tema está obteniendo en España un alto nivel de elaboración teórica en el campo jurídico (E. Sánchez Jiménez, 199910; V. Méndez Baiges, $2002^{11}$ ). Una encuesta del Centro de Investigaciones Sociológicas (CIS, 1992), que es uno de los estudios más recientes sobre este tema del que se puede disponer, obtuvo los resultados que a continuación se detallan (tablas 5 y 6).

TABLA 5

Opiniones sobre la muerte

Sí
¿Cree que, con independencia de su edad, la vida de una per- sona enferma debería prolongarse artificialmente cuando no hay posibilidades de cura?
¿Los médicos deberían dar medicinas para aliviar el dolor de los enfermos incurables, incluso si con ello acortan la vida del paciente?

Un enfermo incurable con grandes dolores, ¿tiene derecho a recibir de los médicos algún producto que termine con su vida sin sufrimiento?

En estos casos, ¿la ley debería permitir a los médicos que pusieran fin a su vida y a su dolor en el caso de que el paciente lo pidiese?

En caso de que el paciente no pueda hacerlo por sí mismo y lo hagan por él sus familiares, ¿debería permitirlo la ley?
No No sabe No contesta
11

1

8

1

28

12

1

FUENTE:

Centro de Investigaciones Sociológicas, Encuesta n. ${ }^{\circ}$ 1996, 2.492 entrevistas, marzo 1992, población en general mayor de 18 años.

10 E. Sánchez Jiménez (1999): La eutanasia ante la moral y el derecho, Universidad de Sevilla, Secretariado de Publicaciones, Sevilla.

11 V. Méndez Baiges (2002): Sobre morir: eutanasia, derechos, razones, Editorial Trotta, Madrid. 
TABLA 6

Conducta personal en algunos casos de enfermedades terminales «En caso de que un miembro de su familia sufriese una enfermedad incurable y muy dolorosa, y pidiese repetidamente que le ayudase a morir, ¿cuál de estas decisiones estaría dispuesto a adoptar y cuáles no?»

Sí

A. Pediría a los médicos que le diesen suficientes analgésicos para calmar el dolor, incluso si con ello acelerase el fin de su vida

B. Pediría a los médicos que interviniesen para poner fin a su vida

C. Si fuese necesario, yo contribuiría personalmente a acelerar el fin de su vida

D. Me opondría a cualquier medicina o tratamiento que pudiese acortar su vida
74

44

24

24

No No sabe No contesta

FUENTE:

Centro de Investigaciones Sociológicas, Encuesta n. ${ }^{\circ}$ 1996, 2.492 entrevistas, marzo 1992, población mayor de 18 años.

Por encima del $50 \%$ de las respuestas estaban a favor de la calidad de muerte, de administrar a los enfermos medicinas contra el dolor aunque acortasen su vida, y de no castigar a los doctores que ayudasen a morir a sus pacientes cuando así se lo solicitaban.

Las escasas excepciones a la mayoría provienen de personas con nivel de educación muy bajo, alto nivel de práctica religiosa y una ideología de extrema derecha. Pero, incluso dentro de estos segmentos de la población, estas respuestas no eran frecuentes, ni eran más numerosas que las respuestas a favor de aplicar la tecnología médica el servicio de una muerte más rápida y menos dolorosa.

Otra institución privada de investigación diferente del ya citado Centro de Investigaciones Sociológicas (CIS), que también ha contribuido al conocimiento de la actitud de la población española hacia la muerte, es el Centro de Estudios sobre la Realidad Social (CIRES). En 1992 desarrolló una encuesta sobre «Ética Social» y en 1994 una encuesta sobre «Salud», en las que se incluyeron algunas preguntas que tienen que ver con este tema. Los resultados se muestran en las tablas 7 y 8 . Las opiniones favorables a la eutanasia son ma- 
TABLA 7

Opinión sobre la eutanasia según características socioeconómicas

\begin{tabular}{|c|c|c|c|c|c|c|}
\hline Noviembre 1992 & Total & (1) \% & (2) \% & (3) \% & $\begin{array}{r}\text { No sabe/ } \\
\text { no contesta }\end{array}$ & Total \\
\hline Total & 1.200 & 33 & 43 & 18 & 5 & 100 \\
\hline \multicolumn{7}{|l|}{ Edad } \\
\hline 18 - 29 años & 311 & 41 & 47 & 9 & 3 & 100 \\
\hline 30 - 49 años & 421 & 35 & 47 & 13 & 5 & 100 \\
\hline 50 - 64 años & 272 & 30 & 39 & 26 & 6 & 100 \\
\hline 65 y más & 196 & 20 & 38 & 32 & 10 & 100 \\
\hline \multicolumn{7}{|l|}{ Posición social } \\
\hline Baja & 465 & 30 & 39 & 24 & 8 & 100 \\
\hline Media & 537 & 34 & 45 & 17 & 4 & 100 \\
\hline Alta & 198 & 38 & 49 & 10 & 3 & 100 \\
\hline \multicolumn{7}{|l|}{ Ideología } \\
\hline Izquierda & 370 & 39 & 47 & 11 & 3 & 100 \\
\hline Centro & 144 & 27 & 45 & 24 & 4 & 100 \\
\hline Derecha & 126 & 28 & 44 & 24 & 5 & 100 \\
\hline \multicolumn{7}{|l|}{ Religión } \\
\hline Alta & 344 & 21 & 37 & 32 & 9 & 100 \\
\hline Media & 243 & 27 & 48 & 19 & 6 & 100 \\
\hline Baja & 550 & 40 & 46 & 11 & 3 & 100 \\
\hline
\end{tabular}

(1) Debería permitirse ayudar a morir a las personas que lo piden.

(2) Sólo debería permitirse bajo ciertas circunstancias.

(3) Nunca debería permitirse.

FUENTE:

CIRES, noviembre 1992, Encuesta sobre Ética Social, 1.200 entrevistas a personas mayores de 18 años.

yoritarias en todos los segmentos sociales, tanto en términos absolutos como relativos. Lo contrario sólo sucede, y ligeramente y en términos relativos, entre el segmento de personas de bajo nivel social que sobrepasan los 50 años de edad.

Los datos más recientes sobre eutanasia proceden de una encuesta de ámbito nacional (muestra de 1.204 entrevistas personales, a población mayor de 18 años), llevada a cabo por ASEP (Análisis Sociales, Económicos y Políticos) en 2003, y cuyos resultados inéditos se dan a conocer por primera vez en este trabajo*. Como mejora metodológica y para evi-

\footnotetext{
* Agradezco especialmente a ASEP la cesión gratuita de sus datos para este estudio.
} 
TABLA 8

Actitud que deberían tener los profesionales sanitarios hacia la eutanasia activa y pasiva según condición socioeconómica

\begin{tabular}{|c|c|c|c|c|c|c|}
\hline Febrero 1994 & Total & (1) \% & (2) \% & $\begin{array}{r}\text { Ina } \\
\%\end{array}$ & $\begin{array}{l}\text { be/ } \\
\text { sta } \\
\%\end{array}$ & Total \\
\hline Total & 1.200 & 63 & 27 & 2 & 7 & 100 \\
\hline \multicolumn{7}{|l|}{ Sexo } \\
\hline Varones & 576 & 64 & 27 & 2 & 7 & 100 \\
\hline Mujeres & 624 & 63 & 28 & 2 & 7 & 100 \\
\hline \multicolumn{7}{|l|}{ Edad } \\
\hline 18 - 29 años & 311 & 69 & 23 & 2 & 5 & 100 \\
\hline 30 - 49 años & 421 & 69 & 24 & 2 & 5 & 100 \\
\hline 50 - 64 años & 272 & 55 & 34 & 2 & 9 & 100 \\
\hline 65 y más & 196 & 55 & 33 & 2 & 10 & 100 \\
\hline \multicolumn{7}{|c|}{ Nivel de educación } \\
\hline Bajo & 726 & 59 & 31 & 2 & 9 & 100 \\
\hline Medio & 340 & 73 & 22 & 2 & 2 & 100 \\
\hline Alto & 128 & 67 & 24 & 4 & 5 & 100 \\
\hline \multicolumn{7}{|l|}{ Posición social } \\
\hline Bajo & 507 & 59 & 30 & 2 & 9 & 100 \\
\hline Medio & 525 & 65 & 28 & 2 & 6 & 100 \\
\hline Alto & 168 & 72 & 19 & 4 & 5 & 100 \\
\hline
\end{tabular}

(1) Los profesionales de la salud deberían respetar la voluntad de los pacientes que no deseen continuar viviendo bajo circunstancias extremas.

(2) La obligación de todos los profesionales de la salud es mantener la vida humana, independientemente de cuáles sean las circunstancias.

FUENTE:

CIRES, febrero 1994, Encuesta sobre Salud, 1.200 entrevistas a la población mayor de 18 años.

tar el riesgo de un posible sesgo de identificación con las frases expresadas positiva y afirmativamente respecto a la eutanasia, se introdujeron expresiones que exigiesen también a los entrevistados el esfuerzo de posicionarse negativamente, por ejemplo, en la frase «la eutanasia debería prohibirse en todos los casos».

Los resultados son similares y consistentes con las encuestas anteriormente analizadas, que, como ya se ha señalado, han sido realizadas por diversas instituciones públicas y privadas. 
En ninguno de los grupos (edad, sexo, posición social, ideología, status socieconómico, familiar, tipo de hábitat, grado de exposición a los medios de comunicación social, orientación política, satisfacción con el gobierno o partido votado en las últimas elecciones generales), el índice medio de acuerdo con la opinión expresada en la frase «la eutanasia debería prohibirse en todos los casos» alcanza los cien puntos, que es el punto medio entre el mínimo (cero puntos) y el máximo posible (doscientos puntos) en la escala del índice simple. La puntuación media en el índice simple es 73 puntos. Además del índice simple, hemos realizado dos índices ponderados. El índice ponderado I toma en consideración la intensidad de acuerdo o desacuerdo; el punto medio sería 2,00. En todos los grupos, excepto el minúsculo que dice no recordar a qué partido votó en las pasadas elecciones, se alcanzó esta puntación media. El índice ponderado II expresa la misma idea de otra manera, por el procedimiento de restar los desacuerdos a los acuerdos y ponderar la intensidad de la posición. Todas las categorías alcanzan puntuación negativa (tabla 9).

En resumen, la encuesta refleja una clara y general opinión pública favorable a la eutanasia bajo ciertas circunstancias y el rechazo a su prohibición. 


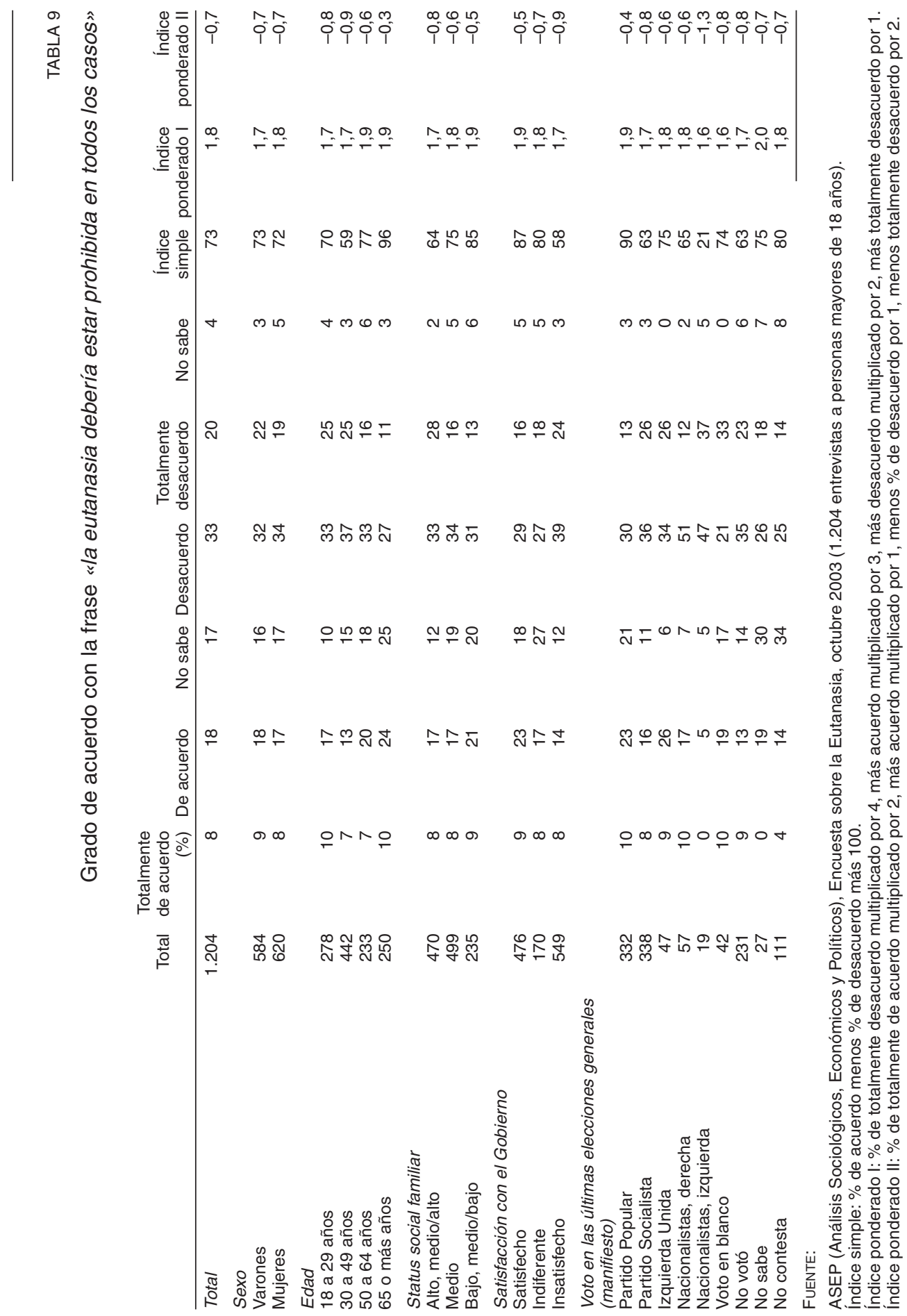




\section{LA OPINIÓN DE LOS JÓVENES}

Otra consulta a la opinión pública, hecha por el Centro de Investigaciones Sociológicas en 2001 entre jóvenes de 15 a 29 años, mostró resultados claramente favorables a la intervención para terminar la vida en ciertas circunstancias. Los principales resultados, que son positivos, se muestran en la tabla 10. Los resultados positivos respecto a la ayuda a morir a otros contrastan con los resultados obtenidos en la misma encuesta respecto al suicidio (no el de los enfermos, sino el general), que fueron bastante negativos (sólo 10,4\% a favor, $82,0 \%$ en contra, $6,2 \%$ no sabe, $1,5 \%$ no contesta).

TABLA 10

La opinión de la juventud

(«Independientemente de lo que usted haría personalmente, ¿está a favor o en contra de ayudar a morir a un enfermo terminal que lo solicita?»)

\begin{tabular}{cccc} 
En contra & A favor & No sabe & No contesta \\
\hline 17,8 & 72,2 & 8,4 & 1,6
\end{tabular}

FUENTE:

Centro de Investigaciones Sociológicas, Encuesta sobre valores y creencias de los jóvenes, 2001. Encuesta n. ${ }^{\circ} 2440 / 0$, pregunta . $^{\circ}$ 12. Muestra de 2.500 entrevistas personales a la población de 15 a 29 años.

En marzo de 2003, en un sondeo conjunto del CIS y del Instituto de la Juventud (n. ${ }^{\circ} 2482$, primera oleada; 1.457 entrevistas a jóvenes de 15 a 29 años), volvió a plantearse una pregunta sobre la eutanasia, aunque en un contexto diferente. Se planteó dentro de un bloque de frases referentes a conductas delictivas de bajo rango, o de clara evaluación negativa por el conjunto de la sociedad española, tales como «romper señales de tráfico», "emborracharse», «hacer trampa en exámenes», «robar artículos en grandes almacenes» o «enfrentarse violentamente a la policía», sobre las que se pedía que los jóvenes manifestaran su grado de adhesión, en una escala de 1 al 10. Ninguna de estas frases llegó a una adhesión media de cuatro puntos. En el mismo bloque, intercaladas, había tres preguntas relacionadas con la muerte: "que se aplique la eutanasia a todo aquel que lo pida» (obtuvo 6,00 puntos de adhesión), «aplicar la pena de muerte» (2,20 puntos) y «que una persona se suicide» (3,02 puntos). Claramente, y a pesar de la negatividad del contexto, los jóvenes se manifestaron mayoritariamente a favor de que se aplique la eutanasia a quien la solicita. 


\section{DE LAS OPINIONES A LA CONDUCTA ACTIVA; LA ACTITUD DE LOS MÉDICOS}

En una sociedad en la que los últimos días de la vida transcurren en ambientes altamente medicalizados, la actitud de los médicos es de importancia crucial para la introducción de cambios relacionados con la calidad de muerte.

No existen datos disponibles sobre la calidad de muerte en los hospitales y otros contextos sanitarios, pero en el año 2002 se realizó una encuesta a médicos acerca de la eutanasia. La encuesta fue patrocinada por el Ministerio de Sanidad y realizada por el Centro de Investigaciones Sociológicas. Hay que destacar que la cooperación al suicidio o la eutanasia es ilegal, por lo que aunque el anonimato se garantice, siempre hay algo de riesgo al responder a ciertas cuestiones.

Muchos médicos, debido a su campo de especialidad, manifiestan que nunca entran en contacto con los enfermos terminales (el 33\% de los entrevistados). Según los resultados de la encuesta, el $79 \%$ de los médicos dijo que en el último año ninguno de sus pacientes le había pedido que le retirase un tratamiento que no le hubiera curado, sino prolongado una enfermedad dolorosa. Con otras palabras, puede suponerse que el resto, un $21 \%$ de los médicos, habría recibido algunas demandas de ayuda; si se excluyen los médicos que dicen que por su especialidad no tienen contacto con enfermos graves, equivale al $33 \%$ de los restantes. Como promedio, e incluyendo todas las especialidades (también las que no suelen tratar enfermos graves), cada médico recibió 3,6 demandas de este tipo el año pasado. Otro 7\% de médicos informó haber recibido peticiones de una intervención más activa (eutanasia activa o suicidio asistido). Cuando se refieren a toda su vida profesional y no sólo al último año, el 58\% de los médicos informa haber recibido peticiones para retirar un tratamiento, $19 \%$ para una sobredosis letal y $8 \%$ ha recibido de algún paciente la petición de alguna medicina letal con el fin de poner fin a su vida por sí mismo.

En el momento actual, en España los médicos no pueden responder positivamente a estas peticiones de los pacientes sin correr un grave riesgo profesional y legal. En una escala de 10 puntos que muestra el grado de acuerdo o desacuerdo con varias frases relativas a las condiciones de muerte, la frase que dice que "administrar a un paciente una dosis letal puede causar serios problemas legales y profesionales al médico» obtuvo el máximo nivel de acuerdo, mientras que otras frases referentes al grado actual de adecuación de los cuidados paliativos (disponibilidad de servicios, apoyo psicológico a las familias) obtuvieron puntuaciones muy bajas. También obtuvieron un nivel muy alto de adhesión las frases «es una práctica médica comúnmente aceptada, aumentar la dosis de analgésicos a pacientes terminales con el fin de aliviar su sufrimiento, aun sabiendo que podría acelerar el fin de sus vidas» (8,43 puntos) y «algunas veces es adecuado retirar o no iniciar un tratamiento a 
pacientes con muy mala calidad de vida» (8,43 puntos). La disparidad de adhesiones ante la frase referente a los riesgos y la referente a los servicios disponibles ilustra bien el conflicto que vive la sociedad española en este tema; desmiente la eficacia de algunas actitudes ocultistas que confían en que, llegada la ocasión, el personal sanitario tomará las medidas oportunas sin ruido y sin necesidad de manifestar abiertamente su conducta. No sólo no existen los servicios paliativos suficientes, sino que las horas finales del enfermo dependen del azar del equipo o personal sanitario al que corresponda atenderle en sus últimas horas; y los sanitarios dependen, asimismo, de la buena o mala voluntad con que sus actos de ayuda al enfermo sean interpretados por sus propios colegas, por los familiares o por otros colectivos sociales.

En opinión de la mayoría de los médicos, un buen sistema de cuidados paliativos podría reducir las demandas de eutanasia, pero no terminaría con ellas. Es mayoría la proporción de médicos que dicen que las leyes actuales deberían cambiarse para facilitar la eutanasia activa o el suicidio asistido ( $41,5 \%$ está de acuerdo en el caso de pacientes terminales en buenas condiciones mentales; $18,4 \%$ también está de acuerdo incluso cuando la enfermedad no es terminal, sino crónica), en tanto que el $31 \%$ se opone al cambio legal y el $9 \%$ no responde.

Todas las iniciativas para mejorar la calidad de vida de los pacientes terminales se puntuaron bien por los médicos, incluida la despenalización de la eutanasia (6,22 puntos de acuerdo como promedio, en una escala máxima de 10 puntos).

\section{EL TESTAMENTO VITAL: LA APROXIMACIÓN DE PERSPECTIVAS DESDE DISTINTAS POSICIONES IDEOLÓGICAS}

No es frecuente que la manifestación de los deseos sobre la propia muerte se haga explícita. El tradicional «testamento» español es un documento de últimas voluntades, pero usualmente su contenido es sólo económico, relativo a la futura distribución del patrimonio que se deja en herencia. La legislación civil ha regulado este campo y es mucho más frecuente que se utilice por la clase alta y la población acomodada que por la clase mediabaja o los estratos de bajo nivel socioeconómico. Sin embargo, en España el 84\% de las familias son propietarias de sus viviendas o alojamientos, y el testamento podría ayudar a clarificar el reparto de la herencia. Según una encuesta del CIS realizada en 1989 (la única encuesta que hemos hallado que ha incorporado esta pregunta), sólo el 18,7\% de la población mayor de 18 años ha otorgado testamento, a lo que cabe añadir un porcentaje similar que espera hacerlo en el futuro. La mayoría de la población $(61,0 \%)$ no ha pensado en hacerlo. Respecto a los que esperan hacerlo en el futuro, un tercio espera hacerlo cuando envejezca, y un $5 \%$ cuando se encuentre enfermo. Por supuesto, muchas personas toman 
decisiones económicas orientadas a compartir o distribuir su patrimonio entre familiares cuando están en buena salud, sin necesidad de un documento formal de últimas voluntades. De estos últimos, la mayoría lo hace «en contra» del Ministerio de Hacienda, con el objetivo más o menos declarado de evitar costes de transmisión o impuestos sobre patrimonio, renta y herencia.

Para muchos españoles, la dimensión económica de la muerte es un tema importante, aunque sensible. El alargamiento de los tratamientos en el caso de las enfermedades terminales tiene un alto coste, tanto en recursos monetarios como no monetarios. Entre otros, tiene un alto coste en salud para los familiares del enfermo grave. Como los recursos son limitados, su agotamiento entra en conflicto con otros objetivos alternativos. Muy a menudo, son los propios enfermos quienes no desean que las metas por las que han luchado toda su vida (por ejemplo, garantizar la educación de sus hijos o proteger a su cónyuge para la época de vejez, o atender a un familiar enfermo o discapacitado) se pongan en peligro por el alargamiento de su propia enfermedad incurable.

Una señal de que los tiempos han cambiado es el «testamento vital», un documento en el que la persona declara cómo desea ser tratada durante su etapa final de enfermedad. Lo que revela el cambio de los tiempos es la solicitud explícita de que «no se prolongue la vida bajo ciertas condiciones" (como fuerte dolor o razonable falta de expectativas de curación). Esta nueva actitud requiere una completa revolución en el sistema sanitario, el abandono de la agónica insistencia en la «lucha contra la muerte» a cualquier precio, bajo cualquier dolor, en todo momento. Lo que - al menos en España - trae esperanza a los pacientes que sufren enfermedades terminales y no encuentran sentido a la prolongación de su situación es que, por primera vez en la historia, dos entidades con raíces ideológicas tan dispares como la católica Conferencia Episcopal y la laica Asociación por el Derecho a Morir Dignamente (DMD) están de acuerdo en el punto esencial de los formularios (documentos para ser firmados por el interesado) que cada cual ha preparado como documento vital y que han sido ampliamente divulgados a través de Internet. Los fundamentos y argumentación esgrimidos en cada formulario son muy diferentes, porque no podría ser de otro modo, ya que la primera se apoya en premisas religiosas y la segunda en humanistas. Pero la esencia es la misma: el reconocimiento de que la calidad de muerte es un derecho humano del que nadie debería despojar a otro, y que la muerte debería ser tan humana y buena como la propia vida.

La Ley General de Sanidad, vigente para todo el territorio español desde mayo de 2003, reconoció el derecho de los pacientes a expresar anticipadamente su voluntad a través de este tipo de testamento y prometió, asimismo, la creación de archivos en que pudiesen de- 
positarse. Muy recientemente, en una experiencia pionera, Andalucía ha abierto el registro de testamentos vitales.

En las elecciones legislativas de marzo de 2004, el partido que posteriormente ganó las elecciones (PSOE) incluyó en su programa electoral la propuesta de crear una Comisión en el Congreso de los Diputados que debatiese sobre «el derecho a la eutanasia y a una muerte digna, los aspectos relativos a su despenalización, el derecho a recibir cuidados paliativos y el desarrollo de tratamientos de dolor».

Por todo ello, cabe esperar que a lo largo de la próxima legislatura se produzcan otras iniciativas legales que, sumadas a la ya mencionada y publicada en el Boletín de las Cortes Generales el 23 de abril de 2004, cambien el panorama de los derechos y prácticas sociales relativos a la muerte en España.

\section{BIBLIOGRAFÍA}

ANSUÁTEGUI ROIG, F. J. (coord.) (1999): Problemas de la eutanasia, Universidad Carlos III de Madrid, Dykinson, Madrid.

CALLAHAN, D. (1996): «El problemático sueño de la vida: en busca de una muerte tranquila», en Morir con dignidad: Dilemas éticos en el final de la vida, Ética y Humanidades, Fundación Ciencias de la Salud, Madrid, pp. 91-105.

CASADO, M., y ROYES, A. (coords.) (2003): Documento sobre la disposición de la propia vida en determinados supuestos: declaración sobre la eutanasia, Observatori de Bioètica i Pret, Parc Científic de Barcelona.

CASTRA, M. (2003): Bien mourir. Sociologie des soins palliatifs, Le Lien social, Presses Universitaires de France, París.

DÍEZ RIPOLLÉS, J. L., y MUÑOZ SÁNCHEZ, J. (coords.) (1996): El tratamiento jurídico de la eutanasia: una perspectiva comparada, Tirant lo Blanch, Valencia.

DURÁN, M. A. (1983): Desigualdad social y enfermedad, Tecnos, Madrid.

- (2003): Los costes invisibles de la enfermedad, Fundación BBVA, Madrid.

- (2003): Diario de Batalla. Mi lucha contra el cáncer, Ediciones Aguilar, Madrid.

— (2003): «El coste de la salud para las familias», revista Arbor, n. ${ }^{\circ} 694$, Madrid.

GONZÁLEZ BARÓN, M. (1996): «La atención del enfermo terminal», en Morir con dignidad: Dilemas éticos en el final de la vida, Ética y Humanidades, Fundación Ciencias de la Salud, Madrid, pp. 53-71.

GRACIA, D. (1996): “Cuestiones de vida o muerte. Dilemas éticos en los confines de la vida», en Morir con dignidad: Dilemas éticos en el final de la vida, Ética y Humanidades, Fundación Ciencias de la Salud, Madrid, pp. 106-140.

MÉNDEZ BAIGES, V. (2002): Sobre morir: eutanasias, derechos, razones, Editorial Trotta, Madrid.

NÚÑEZ PAZ, M. A. (1999): Homicidio consentido, eutanasia y derecho a morir con dignidad, Editorial Tecnos, Madrid. 
PRADO, C. G., y TAYLOR, S. J. (1999): Assisted Suicide: Theory and Practice in Elective Death, Humanity Books, New York.

QUILL, Timothy E. (1996): «La muerte médicamente asistida: ¿progreso o peligro?», en Morir con dignidad: Dilemas éticos en el final de la vida, Ética y Humanidades, Fundación Ciencias de la Salud, Madrid, pp. 14-37.

REICH, Warren T. (1996): El arte de cuidar a los moribundos, Ética y Humanidades, Debates de la Fundación Ciencias de la Salud, Madrid, pp. 71-91.

SÁNCHEZ JIMÉNEZ, E. (1999): La eutanasia ante la moral y el derecho, Universidad de Sevilla, Secretariado de Publicaciones, Sevilla.

TEN HAVE, H. (1996): Eutanasia: la experiencia holandesa, Ética y Humanidades, Debates de la Fundación Ciencias de la Salud, Madrid, pp. 38-53.

THOMASMA, David C., y KUSHNER, T. (eds.) (1999): De la vida a la muerte. Ciencia y bioética, Editorial Cambridge, Madrid.

\section{ESTADÍSTICAS Y ENCUESTAS}

ASEP: Encuesta sobre la eutanasia, octubre 2003.

CIRES: Encuesta sobre Ética Social, noviembre 1992.

CIRES: Encuesta sobre Salud, febrero 1994.

CIS: «Encuesta General de Población» (Nacional), Encuesta n. ${ }^{\circ} 1841$, octubre 1989.

CIS: «Aborto y Eutanasia», Encuesta n. ${ }^{\circ}$ 1996, marzo 1992.

CIS: «Barómetro», Encuesta n. ${ }^{\circ} 2244$, abril 1997.

CIS: «Valores y creencias de los jóvenes», Encuesta n. ${ }^{\circ}$ 2440, diciembre 1997.

CIS: «Encuesta sobre actitudes y valores en las relaciones interpersonales», Encuesta n. ${ }^{\circ} 2442$, enero 2002.

CIS: «Los médicos ante la eutanasia», Encuesta n. ${ }^{\circ} 2451$, abril-mayo 2002.

CIS e Instituto de la Juventud: Encuesta n. ${ }^{\circ} 2482$, marzo 2003.

EUROSTAT: Yearbook, 2002.

EUROSTAT: «Statistiques sociales européennes. Démographie», 2001.

The World Bank: «World Development indicators», 2002. 


\section{ABSTRACT}

The aspiration to a good quality of life prolongs itself in a natural way in the aspiration to a good quality of death. Summarized in this study are a dozen sociological studies carried out in Spain by various institutions throughout the last decade (CIS, CIRES and ASEP, amongst others) on different aspects of death, the fundamental will and euthanasia. The main conclusion is that the opinions and attitudes of Spanish society on this topic are much more open than is acknowledged by prevailing legislation. The subject is of great interest and is a current issue since it appears in the electoral programme of the party that won the elections in March 2004 and because of ongoing legislative proposals.

Key words: Death, Mortality, Euthanasia, Spain. 